\title{
Controlled Release of Doxorubicin from Doxorubicin $/ \gamma$-Polyglutamic Acid Ionic Complex
}

\author{
Bhavik Manocha and Argyrios Margaritis \\ Department of Chemical and Biochemical Engineering, The University of Western Ontario, London, ON, Canada N6A 5B9 \\ Correspondence should be addressed to Argyrios Margaritis, amarg@uwo.ca
}

Received 6 November 2009; Accepted 19 February 2010

Academic Editor: Chao Lin

Copyright (C) 2010 B. Manocha and A. Margaritis. This is an open access article distributed under the Creative Commons Attribution License, which permits unrestricted use, distribution, and reproduction in any medium, provided the original work is properly cited.

\begin{abstract}
Formation of drug/polymer complexes through ionic interactions has proven to be very effective for the controlled release of drugs. The stability of such drug/polymer ionic complexes can be greatly influenced by solution $\mathrm{pH}$ and ionic strength. The aim of the current work was to evaluate the potential of $\gamma$-polyglutamic acid ( $\gamma$-PGA) as a carrier for the anticancer drug, Doxorubicin (DOX). We investigated the formation of ionic complexes between $\gamma$-PGA and DOX using scanning electron microscopy, spectroscopy, thermal analysis, and X-ray diffraction. Our studies demonstrate that DOX specifically interacts with $\gamma$-PGA forming random colloidal aggregates and results in almost $100 \%$ complexation efficiency. In vitro drug release studies illustrated that these complexes were relatively stable at neutral $\mathrm{pH}$ but dissociates slowly under acidic $\mathrm{pH}$ environments, facilitating a $\mathrm{pH}$-triggered release of DOX from the complex. Hydrolytic degradation of $\gamma$-PGA and DOX/ $\gamma$-PGA complex was also evaluated in physiological buffer. In conclusion, these studies clearly showed the feasibility of $\gamma$-PGA to associate cationic drug such as DOX and that is may serve as a new drug carrier for the controlled release of DOX in malignant tissues.
\end{abstract}

\section{Introduction}

The efficacy of cancer chemotherapy is limited by its toxicity to healthy tissues. This is partially attributed to the lack of specificity of anticancer agents for cancerous tissues, and partially to the poor biopharmaceutical properties of the drug [1]. Both these factors together dictate the success of chemotherapy. Most of the anticancer agents are administered as intravenous injections/infusions which lead to an initial burst release and subsequent decay of drug concentrations, below therapeutic levels in blood. The success of controlled or sustained release drug formulations has proved that long-term exposure of ailing tissues to moderate drug concentrations is more beneficial than a pulsed supply of the drug at higher concentrations $[1,2]$. The limitations associated with conventional anticancer drug formulations have led to numerous attempts in developing more effective formulations for chemotherapy [1-3].

Doxorubicin (DOX), an anthracycline antibiotic, is a widely used antineoplastic agent; however, its therapeutic efficacy is limited because of the dose-dependent cardiotoxi- city, myelosuppression $[4,5]$, and development of multidrug resistance [6]. Such adverse side effects associated with conventional formulations of DOX have led researchers to investigate alternative forms of administering DOX, for example, prodrug approach or binding/encapsulation in particulate carriers [7-10].

DOX association to nanoparticulate carriers such as liposomes [11], nanoparticles [12], or micelles [13] has led to its controlled release over extended periods of time, thereby increasing its efficacy and reducing toxic side effects. The majority of attempts to associate DOX to polymeric nanoparticles have used either anionic or neutral polymers, such as polyacrylates [14] or pullulan [15], respectively. Anionic polymers exploit the charge interactions with cationic DOX to achieve high association or complexation efficiencies. Despite of such high association, the DOXloaded nanoparticles exhibit negative zeta potential, which impedes their adhesion to cell membranes and hence their cell uptake. To overcome this limitation, the negatively charged nanoparticles are coated with a cationic polymer/lipid layer [16]. In an alternate approach, Janes et al. 


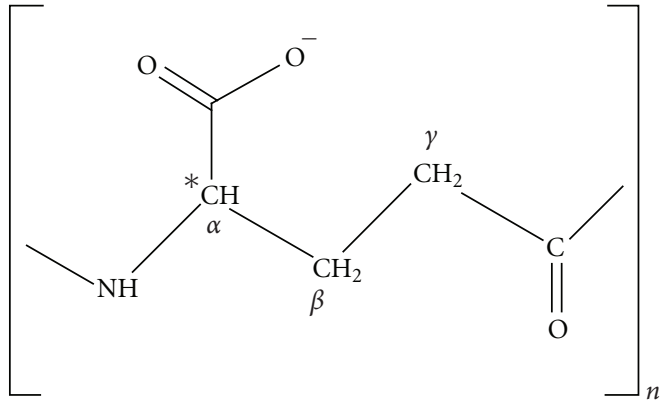

(a)

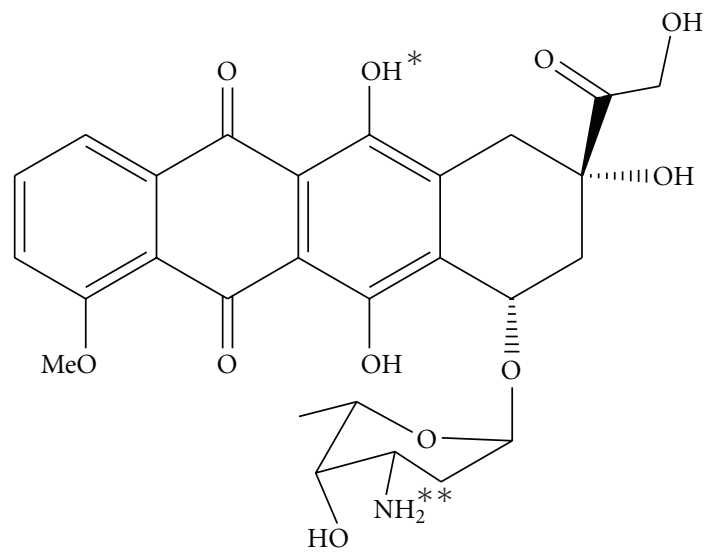

(b)

Figure 1: Chemical structures of (a) $\gamma$-Polyglutamic Acid and (b) Doxorubicin. In (b), * Deprotonable functional group, ** Protonable functional group.

[7] illustrated the ionic complexation of DOX to a positively charged polymeric carrier, chitosan. Although DOX binding to chitosan was very low $(1.4 \% \mathrm{w} / \mathrm{w})$, the DOX-loaded chitosan nanoparticles entered the cell via endocytosis, releasing DOX intracellularly.

Carboxyl groups have the ability to form strong ionic interactions with desired drug candidates because they are excellent hydrogen bond donors [17]. Anionic polymers, owing to their high negative-charge density can bind substantial amounts of cationic drugs. Such an association based on ionic/electrostatic interactions is termed as polymer/drug complexation, and the complex formed is termed as polyelectrolyte complexes. The activity, solubility, cell permeability, and stability of drugs can be manipulated by using polymers with different chemical and physical properties. For instance, $\mathrm{pH}$-induced degradation of basic drug procaine was markedly improved by ion pairing it with acidic polyelectrolyte, carbomer, thereby improving its aqueous chemical stability [18]. Guo et al. [19] demonstrated the controlled release of coenzyme A from $\mathrm{pH}$-sensitive ionic complexes of chitosan/carboxymethyl chitosan. Recently, low molecular weight $\gamma$-PGA was used as an excipient to form complex with a bitter tasting drug, diphenhydramine. The complex not only improved the drug stability and masked its bitter taste, but also facilitated its sustained drug release [20].
Gamma-polyglutamic acid ( $\gamma$-PGA) is a homopolyamide of glutamic acid monomers, connected by amide linkages between $\alpha$-amino and $\gamma$-carboxyl groups (Figure 1(a)). The presence of a free $\alpha$-carboxylate anion per glutamic acid monomer imparts a high anionic character to $\gamma$-PGA, making it a potential candidate to achieve high association efficiencies with cationic drugs. These side-chain carboxyl groups also offer attachment points for the conjugation of chemotherapeutic agents to the polymeric carrier [21]. $\gamma$ PGA is produced as a capsular material or slime by several Bacillus species and is a water-soluble, biodegradable, and biocompatible polymer. $\gamma$-PGA is nontoxic and nonimmunogenic and can be easily assimilated in vivo, rendering it a potential biomaterial for various drug delivery applications, such as nanoparticulate drug/gene carriers, hydrogels, and tissue scaffolds $[3,22,23]$.

The aim of this work was to investigate the use of carboxyl-group-containing polymer, $\gamma$-PGA, to form ionic complexes with a cationic anticancer drug, DOX. The specific interactions between $\gamma$-PGA and DOX to form stable ionic complexes were investigated. The $\mathrm{pH}$-controlled release of DOX from DOX/ $\gamma$-PGA complex and hydrolytic degradation of polymer and drug/polymer complex were also investigated in vitro.

\section{Materials and Methods}

2.1. Materials. Gamma-polyglutamic acid (HM-form; $125 \mathrm{kDa}$ by GPC) was a gift from Natto Biosciences (Montreal, Canada). Doxorubicin hydrochloride was obtained as a $2 \mathrm{mg} / \mathrm{mL}$ solution $(\mathrm{pH} 3.0)$ in $0.9 \%(\mathrm{w} / \mathrm{v})$ sodium chloride from Novopharm (Toronto, Canada). Unless otherwise mentioned, all other chemicals were reagent grade and purchased from Sigma Aldrich (Toronto, Canada). All the glasswares used were pre-treated with SylonCT $(5 \%$ dimethyldichlorosilane in toluene; Sigma Aldrich, Toronto, Canada).

2.2. Formation of $D O X / \gamma-P G A$ Complex. Predetermined amounts of DOX were added to aqueous solutions of $\gamma$-PGA (10 mg/mL; pH 7, nonbuffered) to obtain six different DOX: $\gamma$-PGA weight ratios $(1: 200,1: 100,1: 10$, $1: 1,2: 1$, and $3: 1)$. The DOX/ $\gamma$-PGA mixtures were left under magnetic stirring at room temperature for 24 hours, dialyzed against deionized water $(500 \mathrm{~mL})$ for 24 hours (Spectrapor membrane tubing; MWCO 6-8 kDa, Spectrum Labs, CA, USA), and then freeze-dried overnight. DOX $/ \gamma$ PGA complexes that showed instantaneous precipitation were collected by centrifugation $(11,000 \times \mathrm{g}$ for 10 minutes), washed twice with deionized water, and then freeze-dried.

All the procedures were performed in triplicates. Photodegradation of DOX was prevented by conducting all experiments in dark.

2.3. Evaluation of DOX Complexation with $\gamma$-PGA. DOX $/ \gamma$ PGA complexation efficiency was determined by analyzing the unbound DOX in the dialysate, supernatant, and sample washes using HPLC, as described previously [24]. 
The samples were eluted through Zorbax C18 $(250 \mathrm{~mm}$ $\times 4.6 \mathrm{~mm}, 5 \mu \mathrm{m}$ ) column (Agilent Technologies, Mississauga $\mathrm{ON}$ ), attached to fluorescence detector. The emission and excitation wavelengths were set at 480 and $560 \mathrm{~nm}$, respectively. The mobile phase composition was 70:30 water/acetonitrile with $0.1 \%$ triethylamine; $\mathrm{pH}$ was adjusted to 3.0 with phosphoric acid. The samples were eluted under isocratic conditions at flow rate $1.2 \mathrm{~mL} / \mathrm{min}$ and column temperature $35^{\circ} \mathrm{C}$.

DOX $/ \gamma$-PGA complexation efficiency was calculated as follows:

Dox Encapsulation Efficiency (\%)

$$
=\frac{\text { Total DOX }- \text { Free DOX }}{\text { Total DOX }} \times 100 .
$$

\subsection{Physical Characterization of DOX/ $\gamma$-PGA Complex}

2.4.1. Spectrophotometric Analysis. Doxorubicin concentration was maintained constant at $40 \mu \mathrm{g} / \mathrm{mL}$, and spectra of DOX in the absence and presence of $\gamma$-PGA were recorded from 190 to $600 \mathrm{~nm}$ using UV-Visible spectrophotometer (Cary 50 Conc, Varian, USA) with a $2 \mathrm{~nm}$ slit width and a $1 \mathrm{~cm}$ path length at intervals of $1 \mathrm{~nm}$, using water as the baseline reference.

2.4.2. Size and Surface Characteristics of DOX/ $\gamma$-PGA Complexes. The surface morphology of DOX/ $\gamma$-PGA complexes was examined using a scanning electron microscope (SEM; Hitachi S-3400N, Toronto, ON). A current of $99 \mu \mathrm{A}$, voltage of $15 \mathrm{kV}$, and a working distance of $9.3 \mathrm{~mm}$ were applied. The samples were prepared by placing $5-10 \mu \mathrm{L}$ of precipitates (diluted 50-100×) on formvar grids and allowed to air-dry overnight under vacuum. All samples were sputtered with $\mathrm{Au} / \mathrm{Pd}(10-12 \mathrm{~nm}$ thickness) and consecutive magnifications $(1500 \times, 2000 \times$, and $5000 \times)$ were used to view the morphology of formed DOX $/ \gamma$-PGA complexes.

Particle size and zeta potential measurements of the precipitates were performed by photon correlation spectroscopy (90Plus/BI-MAS) and ZetaPALS Zeta potential analyzer (Brookhaven Instruments, NY), respectively. For size measurements, samples were diluted in MiliQ water and measured for at least 120 seconds. For zeta potential measurements, samples were diluted in $10 \mathrm{mM} \mathrm{KNO}_{3}$. All the measurements were performed in triplicate.

2.4.3. Thermal Analysis. The differential scanning calorimetry (DSC) thermogram of DOX $/ \gamma$-PGA complex was recorded uisng DSC 822e (Mettler Toledo, Columbus, $\mathrm{OH}$ ) and compared with the thermograms of pure DOX and $\gamma$ PGA. The scanning rate was controlled at $10^{\circ} \mathrm{C} / \mathrm{min}$; the starting and ending temperature were 25 and $500^{\circ} \mathrm{C}$, respectively. Thermogravimetric analysis (TGA) was performed using TGA/SDTA 851e (Mettler Toledo, Columbus, OH) with a starting and ending temperatures of 25 and $500^{\circ} \mathrm{C}$, respectively.
2.4.4. X-Ray Diffraction Crystallography. The X-ray crystallographic patterns of DOX, $\gamma$-PGA, and DOX/ $\gamma$-PGA complexes were studied using an X-ray diffractometer (Rigaku, Tokyo, Japan). Samples were pressed into circular form on a quartz sample holder, and scanned from 0 to $60^{\circ}$. A Bragg angle of $2 \theta$ was recorded at a scan rate of $1.0^{\circ} / \mathrm{min}$.

2.4.5. Evaluation of In Vitro DOX Release from $D O X / \gamma$ $P G A$ Complex. Effect of ionic strength and $\mathrm{pH}$ on drug release was studied in different buffers. DOX/ $\gamma$-PGA complex $(1: 1 \mathrm{w} / \mathrm{w})$ was placed in dialysis membrane (Spectrapor membrane tubing; MWCO 6-8 kDa, Spectrum Labs, CA, USA) and immersed in $100 \mathrm{~mL}$ of different buffers of varying $\mathrm{pHs}(154 \mathrm{mM}$ sodium chloride, $\mathrm{pH} 2.2 ; 100 \mathrm{mM}$ acetate buffers $\mathrm{pH} 4.0$ and $6.0 ; 100 \mathrm{mM}$ phosphate buffer saline, pH 7.4), and ionic strengths (77, 154 and $308 \mathrm{mM}$ phosphate buffered saline, $\mathrm{pH}$ 7.4). All flasks were incubated at room temperature under mild agitation. Samples were collected at different time intervals. Buffers in all the flasks were replaced every 24 hour. The free DOX released into the dialysate was estimated using fluorescence HPLC and plotted as a function of time to determine drug release kinetics.

2.4.6. Hydrolytic Degradation Studies. A predetermined amounts of $\gamma$-PGA and DOX $/ \gamma$-PGA ionic complex $(1: 10$ $\mathrm{w} / \mathrm{w})$ were dissolved in $154 \mathrm{mM}$ phosphate buffer saline, $\mathrm{pH}$ 7.4. At specified time intervals, samples were collected and molecular weights were measured by gel permeation chromatography (GPC). The GPC system comprised of Agilent 1200 HPLC system, equipped with two Waters Ultrahydrogel Linear columns in series, a refractive index detector, a UV detector $(210 \mathrm{~nm})$, and a column thermostat maintained at $30^{\circ} \mathrm{C}$. Phosphate buffer $(100 \mathrm{mM}, \mathrm{pH} 7.4)$ was used as a mobile phase at a flow rate of $0.8 \mathrm{~mL} / \mathrm{min}$. The injection volume of all the samples was maintained constant at $25 \mu \mathrm{L}$. Molecular weight calibration was performed with polyethylene glycol/poly (ethylene oxide) standard kit ( $\mathrm{M}_{\mathrm{p}}$ 500-2,740,000) from Sigma-Aldrich, Canada.

The weight average molecular weight $(\mathrm{Mw})$ of polymer was plotted as a function of time to estimate the polymer degradation kinetics.

\section{Results and Discussion}

The main aim of this work was to develop $\gamma$-PGA-based colloidal carrier for anticancer drug, DOX. Because of high negative charge density of $\gamma$-PGA, we expected a fairly high association of DOX owing to electrostatic attraction between the polymer and drug.

DOX is predominantly positively charged amphoteric drug, containing protonable amino group in the sugar moiety and two deprotonable phenolic groups in the aglycone part of the molecule (Figure 1(b)). To begin, we tested the extent of DOX: $\gamma$-PGA association by incubating DOX and $\gamma$-PGA aqueous solutions in different drug/polymer weight ratios, then dialyzing the drug/polymer solution to remove 


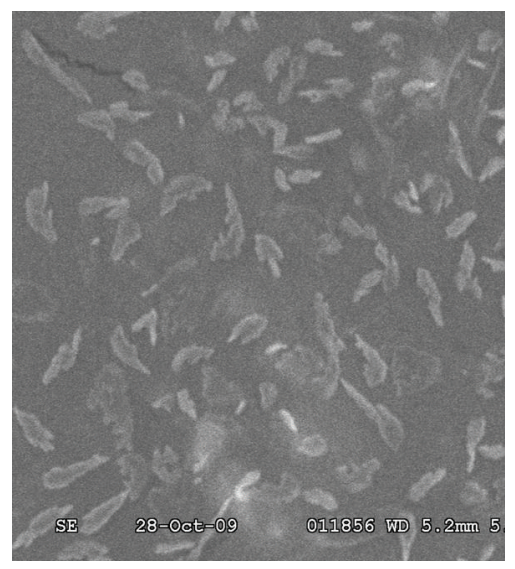

(a)

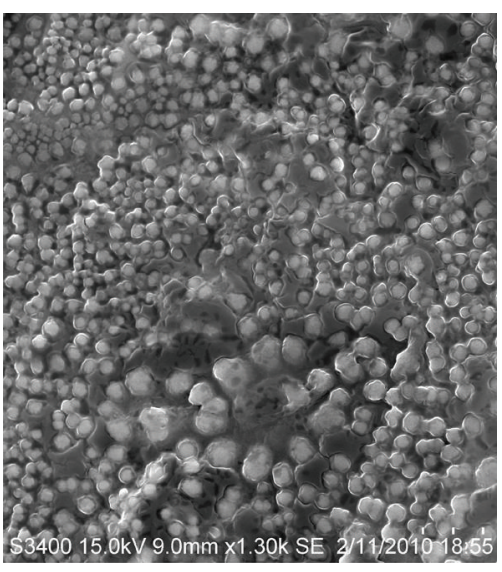

(b)

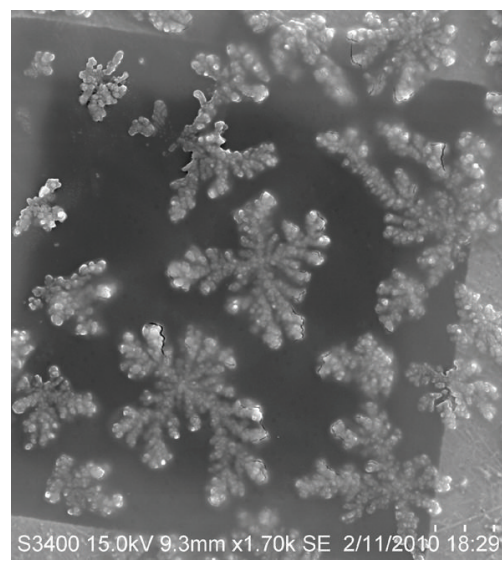

(c)

Figure 2: SEM images of DOX/ $\gamma$-PGA complexes at different DOX: PGA weight ratios: (a) $1: 100 \mathrm{w} / \mathrm{w}(12000 \times$;); (b) $1: 10 \mathrm{w} / \mathrm{w}$ (7000×); (c) $1: 1 \mathrm{w} / \mathrm{w}(10000 \times)$.

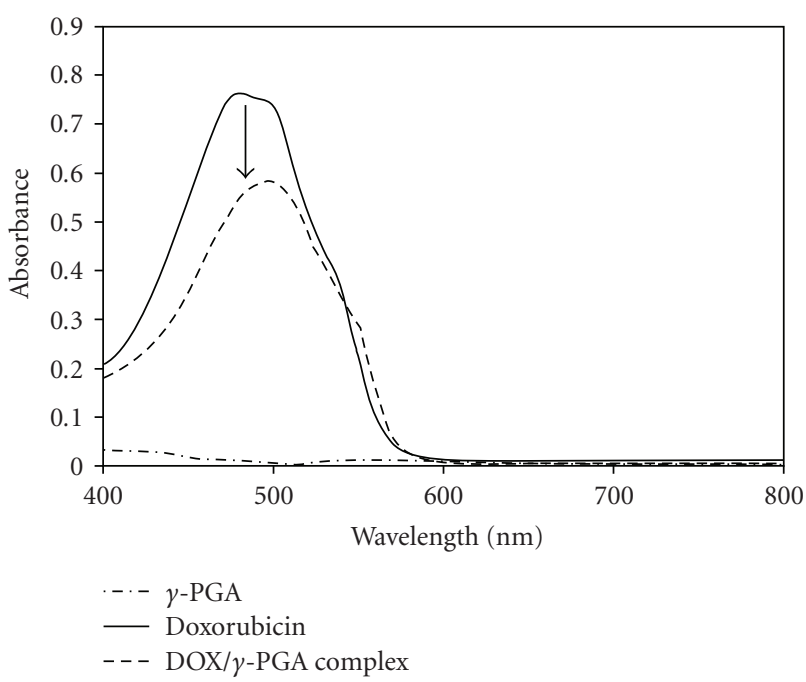

FIGURE 3: Visible spectra of DOX solution, $\gamma$-PGA solution, and $\mathrm{DOX} / \gamma$-PGA complex.

nonassociated DOX, and lyophilizing to promote polymerdrug interactions. For all the DOX: $\gamma$-PGA weight ratios tested, all of the DOX that was added formed complex with $\gamma$-PGA, giving the complexation efficiency of almost $100 \%$ (Table 1). However, a marked difference in the aqueous solubility of various DOX/ $\gamma$-PGA complexes was observed. At lower DOX: $\gamma$-PGA weight ratios, no precipitation was observed during the 24-hour reaction time, and the freezedried complex was easily dissolved in water. For DOX: $\gamma$ PGA 1:1, mild precipitation was observed at the beginning of the reaction; after 24 hours, the complex was collected as a precipitate. The freeze dried 1:1 complex was soluble in water after stirring overnight. In case of $2: 1$ and $3: 1$ $\mathrm{DOX} / \gamma$-PGA complexes, water insoluble precipitates were formed.
TABle 1: Complexation efficiencies for DOX $/ \gamma$-PGA complexes formed by mixing DOX and PGA in different weight ratios $(n=$ 3).

\begin{tabular}{lcc}
\hline $\begin{array}{l}\text { DOX }: \boldsymbol{\gamma} \text {-PGA ratio } \\
(\mathrm{w} / \mathrm{w})\end{array}$ & $\begin{array}{c}\text { Complexation efficiency } \\
(\%)\end{array}$ & $\begin{array}{c}\text { Aqueous } \\
\text { solubility }\end{array}$ \\
\hline $1: 100$ & $99 \pm 0.89$ & Soluble \\
$1: 50$ & $99.3 \pm 1.2$ & Soluble \\
$1: 10$ & $99.7 \pm 2.65$ & Soluble \\
$1: 1$ & $99.1 \pm 0.6$ & Precipitate \\
$2: 1$ & $95.4 \pm 4.1$ & Precipitate \\
$3: 1$ & $96 \pm 6.5$ & Precipitate \\
\hline
\end{tabular}

3.1. Size and Surface Characteristics of DOX/ $\gamma-P G A$ Complexes. SEM micrographs revealed that the formed complexes are submicron-sized random-shaped structures, ranging from elongated needles (Figure 2(a)) to colloidal aggregates (Figures 2(b) and 2(c)). Table 2 shows the size and surface characteristics of various DOX $/ \gamma$-PGA complexes, as measured by particle size and zeta potential analyzer. The average particle size of the precipitates was found to be $\sim 80 \mu \mathrm{m}$. Various DOX/ $\gamma$-PGA ionic complexes exhibited significantly high negative zeta potential values, and zeta potential of complex decreased with the increase in DOX ratio w.r.t. $\gamma$-PGA. As mentioned above, previous studies have shown that nanoparticles with negative zeta potential are most likely rejected by cells owing to charge repulsions between negatively charged cell membranes and particles with negative surface charges. We would like to clarify that DOX $/ \gamma$-PGA complexes are not our final intended formulation.

More studies are underway to overcome the negative zeta potential by formulating the DOX $/ \gamma$-PGA ionic complexes into polymeric nanoparticles of 100-200 nm diameter. 
TABLE 2: Size and surface characteristics of different DOX/ $\gamma$-PGA ionic complexes $(n=3)$.

\begin{tabular}{lccc}
\hline $\begin{array}{l}\text { DOX }: \gamma \text {-PGA } \\
\text { ratio }(\mathrm{w} / \mathrm{w})\end{array}$ & $\begin{array}{c}\text { Particle size } \\
(\mu \mathrm{m})\end{array}$ & Polydispersity & $\begin{array}{c}\text { Zeta potential } \\
(\mathrm{mV})\end{array}$ \\
\hline $1: 200$ & $83 \pm 14$ & $0.45 \pm 0.13$ & $-30.6 \pm 3.1$ \\
$1: 100$ & $82.5 \pm 28$ & $0.45 \pm 0.14$ & $-39 \pm 3.5$ \\
$1: 10$ & $81 \pm 11$ & $0.39 \pm 0.1$ & $-47.7 \pm 2.8$ \\
$1: 1$ & $80 \pm 37$ & $0.53 \pm 0.14$ & $-53.7 \pm 1.1$ \\
\hline
\end{tabular}

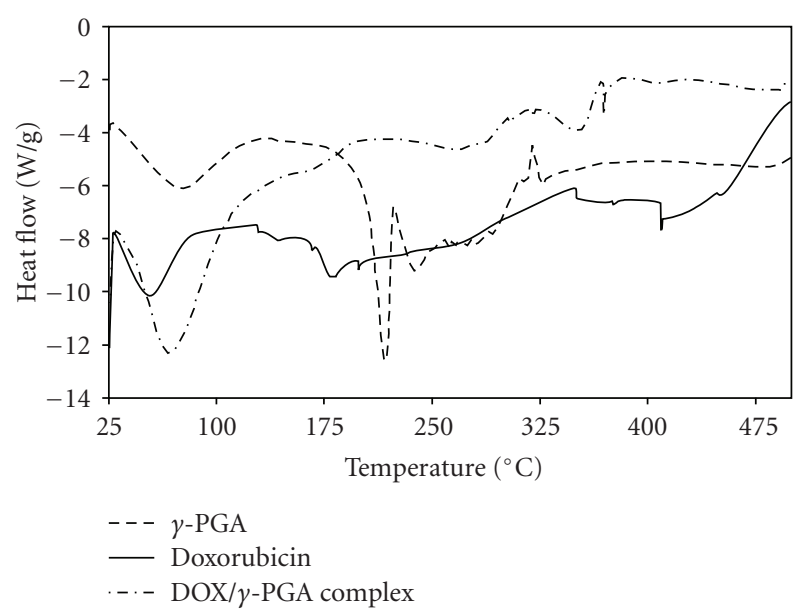

FIGURE 4: DSC thermograms of freeze dried Doxorubicin, $\gamma$-PGA and DOX/ $\gamma$-PGA $(1: 1 \mathrm{w} / \mathrm{w})$ complex.

3.2. Specific Interaction between DOX and $\gamma$-PGA. The interaction of DOX with $\gamma$-PGA was investigated spectrophotometrically by comparing the visible spectra of DOX $/ \gamma$ PGA complex with that of pure $\gamma$-PGA and pure DOX. As shown in Figure 3, DOX has a characteristic $\lambda_{\max }$ at $480 \mathrm{~nm}$; no detectable absorbance was noted for $\gamma$-PGA over the chosen wavelength range at the concentrations tested. In case of DOX/ $\gamma$-PGA complex, spectral changes in DOX peak maxima were observed. The $\lambda_{\max }$ of complex appeared at $495 \mathrm{~nm}$, suggesting that addition of $\gamma$-PGA to DOX induces red-shift of $15 \mathrm{~nm}$. Moreover, the intensity of DOX peak at $480 \mathrm{~nm}$ was reduced by $\sim 23 \%$ which suggest that a complex was formed between $\gamma$-PGA and DOX. No significant difference in $\mathrm{pH}$ was observed upon mixing $\gamma$ PGA and DOX solutions.

As stated above, $\gamma$-PGA exhibits high negative character because of the presence of one carboxylate anion per monomer (Figure $1(\mathrm{a})$ ). The $\mathrm{pK}_{\mathrm{a}}$ of $\gamma$-PGA is 2.6 (as calculated by acid-base titration), thus in slightly acidic to neutral environment, the $\alpha$-carboxyl groups remain unprotonated. Doxorubicin is an amphoteric drug containing protonable amino group and deprotonable phenolic groups; thus a continuous equilibrium exists between the positively charged, negatively charged, neutral and zwitter ionic species of DOX. In the $\mathrm{pH}$ range of $0-6$, the amine group in DOX is protonated and exists in the form of $\mathrm{NH}_{3}^{+}$. Thus, it is most likely that the electrostatic interactions between negatively charged $\gamma$-PGA and positively charged DOX lead

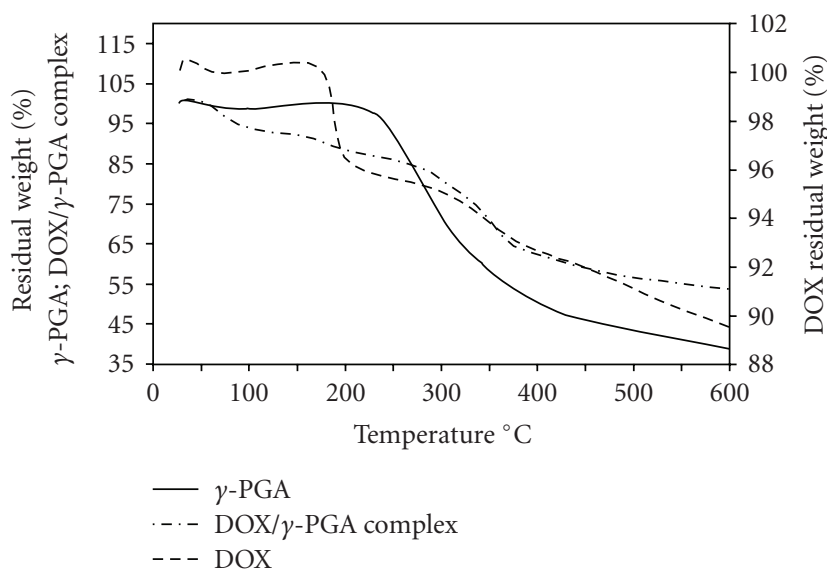

FIGURE 5: TGA thermograms of DOX, $\gamma$-PGA, and DOX/ $\gamma$-PGA $(1: 1 \mathrm{w} / \mathrm{w})$ complex.

to the formation of DOX $/ \gamma$-PGA complex. In a similar study, Agresti et al. [20] reported the formation of an ionic complex between PGA and diphenhydramine (DPH) under acidic conditions ( $\mathrm{pH}$ 3.0). The DPH/PGA complex was formed by $\mathrm{pH}$-induced transition in PGA conformation from random coil to $\alpha$-helix. DPH was released from the complex under weakly acidic or neutral conditions, resulting in the reversal of PGA conformation from $\alpha$-helix to random coil. In the present work, when aqueous $\gamma$-PGA ( $\mathrm{pH} 7$, nonbuffered; random coil conformation) was mixed with DOX ( $\mathrm{pH} 3.0$, as supplied), the $\mathrm{pH}$ of the mixture was $\sim 6$. As expected, we did not observe any change in $\gamma$ PGA conformation as confirmed by $\mathrm{CD}$ spectral analysis of $\gamma$-PGA and DOX/ $\gamma$-PGA complex at $\mathrm{pH} 6$ and 7 (data not shown). Hence, it can be concluded that electrostatic interaction is the predominant force between DOX and $\gamma$-PGA. However, one cannot rule out the possibility of other factors such as hydrophilic/hydrophobic interactions, resonance effect, and so forth, which might play a role in DOX complexation with $\gamma$-PGA. To further elucidate the type of interaction between DOX and $\gamma$-PGA, DOX $/ \gamma$-PGA complexes were characterized by thermal analysis and X-ray powder diffraction.

\subsection{Characterization of $D O X / \gamma-P G A$ Complexes}

3.3.1. Differential Scanning Calorimetry (DSC). DSC measurements were done to (a) exclude the possibility of pure DOX being precipitated during DOX/ $\gamma$-PGA complexation and (b) to confirm the specific interactions between DOX and $\gamma$-PGA to form stable complexes. The DSC thermograms were plotted simultaneously to compare the thermal properties of newly formed DOX $/ \gamma$-PGA complexes with those of pure DOX and pure $\gamma$-PGA. As illustrated in Figure 4, $\gamma$-PGA exhibited two endothermic peaks at 82 and $218^{\circ} \mathrm{C}$, and an exothermic peak at $320^{\circ} \mathrm{C}$. $\gamma$-PGA obtained from different sources has a varying molecular weight and associated physico-chemical properties such as viscosity, thermal characteristics, and so forth. Very few reports are 


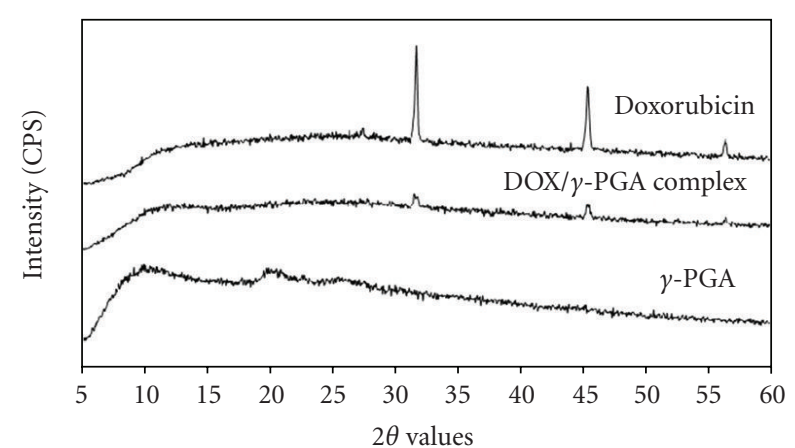

Figure 6: Wide angle X-ray diffraction (WAXS) patterns of DOX, $\gamma$-PGA, and DOX/ $\gamma$-PGA $(1: 1 \mathrm{w} / \mathrm{w})$ complex.

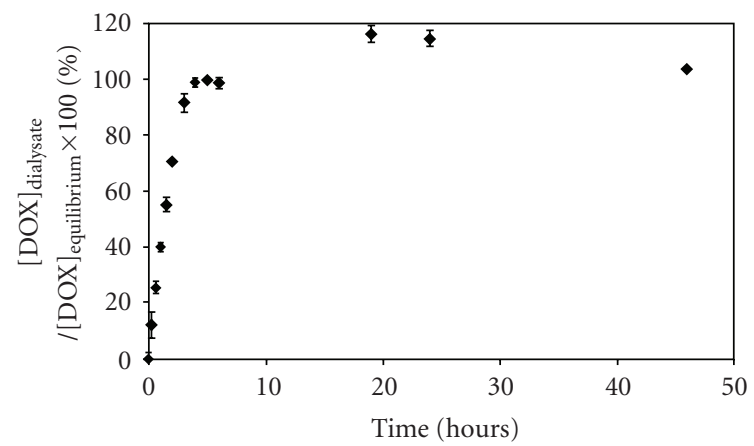

FIgure 7: Control equilibration study of free DOX through the dialysis membrane (MWCO: $6-8 \mathrm{kDa}$ ) used in in vitro release studies. The equilibration is complete after 5 hours. Mean and S.D. are shown $(n=3)$.

available on the glass transition $\left(T_{g}\right)$ and melting $\left(T_{m}\right)$, or decomposition $\left(T_{d}\right)$ temperatures of $\gamma$-PGA. Khachatoorian et al. [25] reported the $T_{g}$ and $T_{m}$ of $\gamma$-PGA to be around $110^{\circ} \mathrm{C}$ and $397^{\circ} \mathrm{C}$; however, the source and molecular weight of $\gamma$-PGA used in their study was not mentioned. Ho et al. [26] reported the thermal decomposition of $\gamma$ PGA H-form (M.W.: $2 \times 10^{6} \mathrm{Da}$ ) obtained from B. subtilis (natto): $T_{m}\left(206^{\circ} \mathrm{C}\right)$ and $T_{d}\left(209.8^{\circ} \mathrm{C}\right)$ [26]. Since $\gamma$-PGA used in this study was obtained from natto gum (M.W: $\sim 127 \mathrm{kDa}, \mathrm{GPC}$ ), thus the first endothermic peak at $82^{\circ} \mathrm{C}$ most likely corresponds to the $T_{g}$ of $\gamma$-PGA, while the sharp endothermic peak at $218^{\circ} \mathrm{C}$ is the melting endotherm $\left(T_{m}\right.$ or $T_{d}$ ) of $\gamma$-PGA.

For DSC of pure drug, DOX from the injection vial was first freeze-dried to obtain a dry powder. Three endotherm peaks appeared at 54,195 , and $355^{\circ} \mathrm{C}$. The sharp endothermic peak at $54^{\circ} \mathrm{C}$ is most likely because of residual moisture left after freeze drying. The peak at $195^{\circ} \mathrm{C}$ corresponds to the melting endotherm of $\operatorname{DOX}\left(T_{m}=218^{\circ} \mathrm{C}\right)[12]$.

In case of DOX $/ \gamma$-PGA complex, a broad endotherm peak appeared at $68^{\circ} \mathrm{C}$ which could possibly be the $T_{g}$ of the complex, since association with DOX may have altered the $T_{g}$ of pure $\gamma$-PGA. The absence of a characteristic melting peak of DOX between 150 and $260^{\circ} \mathrm{C}$ confirms that no pure DOX was precipitated during DOX $/ \gamma$-PGA complexation and that the entire added drug was bound to the polymer.

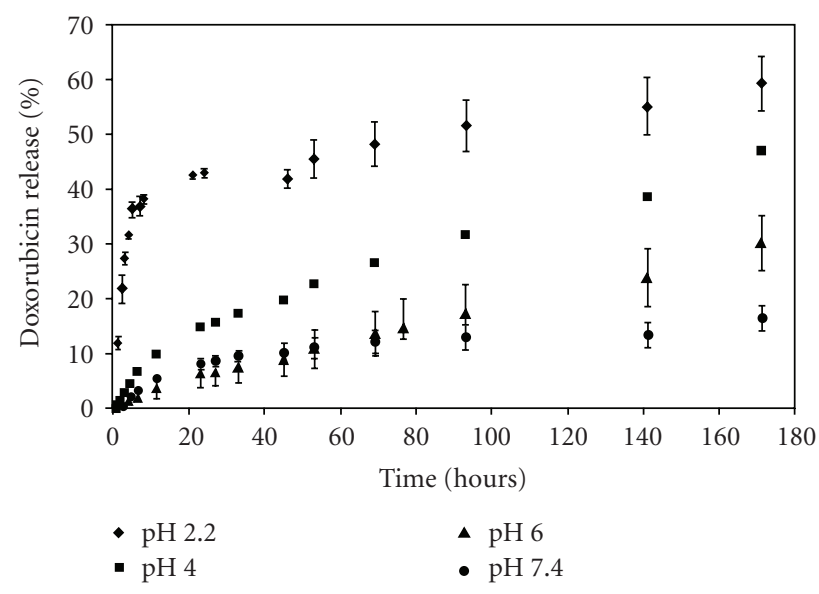

FIGURE 8: In vitro release of bound DOX from DOX/ $\gamma$-PGA complexes $(1: 1 \mathrm{w} / \mathrm{w})$ from dialysis membrane in buffered aqueous solutions at different $\mathrm{pHs}$.

This also strongly indicates the formation of stable DOX/ $\gamma$ PGA complex. An endothermic peak at $360^{\circ} \mathrm{C}$ most likely represents the decomposition of DOX/ $\gamma$-PGA complex.

3.3.2. Thermogravimetric Analysis (TGA). To further evaluate the stability of DOX $/ \gamma$-PGA complex, pure polymer, pure drug, and the polymer/drug complex were analyzed by thermogravimetry. As shown in Figure 5, a total of $10 \%$ weight loss of pure DOX was observed in the tested temperature range from 25 to $600^{\circ} \mathrm{C}$, suggesting that most of the DOX did not decompose to vapor state, and was collected as char in the crucible. The temperature range of $175-220^{\circ} \mathrm{C}$ accounted for up to $4 \%$ loss in DOX weight. This thermal decomposition range matches with the DOX melting peak at $195^{\circ} \mathrm{C}$, as determined by DSC. Thermal degradation of pure $\gamma$-PGA started at $210^{\circ} \mathrm{C}$, which is the $T_{m}$ of $\gamma$-PGA as noted from DSC. Around $60 \%$ of $\gamma$ PGA were decomposed as the temperature reached up to $600^{\circ} \mathrm{C}$. In case of $\mathrm{DOX} / \gamma$-PGA complex, around $60 \%$ of the complex remained undecomposed when heated from 25 to $600^{\circ} \mathrm{C}$, suggesting that DOX is strongly associated to $\gamma$-PGA. The observed weight loss in DOX/ $\gamma$-PGA complex is most likely attributed to thermal decomposition of $\gamma$ PGA. The TGA curve of DOX/ $\gamma$-PGA complex reflects the heterogeneous nature of the interaction between DOX and $\gamma$ PGA. Therefore, it might be suggested that in addition to the possible electrostatic interactions between the amino group of DOX and the carboxyl groups in $\gamma$-PGA, the anthracycline ring of DOX might also be participating in DOX/ $\gamma$-PGA complexation by interacting with the hydrophobic domains on $\gamma$-PGA backbone.

3.3.3. X-Ray Powder Diffraction Analysis. To further elucidate the interaction between DOX and $\gamma$-PGA, the crystal characteristics of the DOX/ $\gamma$-PGA complex were examined using wide angle $\mathrm{X}$-ray powder diffraction (Figure 6). For DOX, clear peaks are visible in the diffractogram indicating the presence of crystalline phase in the native form, whereas 
$\gamma$-PGA showed a typical amorphous pattern. The appearance of identical peaks in the diffractogram of DOX $/ \gamma$-PGA complex confirms the association of DOX with $\gamma$-PGA and that the formed complexes are crystalline in nature.

3.3.4. Drug Release from $D O X / \gamma$-PGA Complexes. Of all the water-soluble DOX/ $\gamma$-PGA complexes that were formed, the one with maximum DOX amount $(1: 1 \mathrm{w} / \mathrm{w}$ DOX: $\gamma$ PGA) was chosen for drug release studies. We simulated "sink" conditions by placing the DOX/ $\gamma$-PGA complex in a dialysis bag (MWCO: 6-8 $\mathrm{kDa}$ ), and regularly replacing the dialysate buffer every 24 hour. A control experiment with free DOX (Figure 7) confirmed that the high MWCO (6$8 \mathrm{kDa}$ ) of dialysis membrane selected ensured unrestricted diffusion of the released drug; the time interval between dialyzate replacement was in excess for reaching the complete equilibration of DOX solutions on either sides of dialysis membrane (5 hours). The highest concentration of drug in the dialyzate was $1.2 \mu \mathrm{g} / \mathrm{mL}$; a value much below $10 \%$ of drug's aqueous solubility, which is a requirement for the fulfillment of "sink" conditions.

Because of predominantly ionic nature of DOX/ $\gamma$-PGA complex, it was expected that changing the $\mathrm{pH}$ and/or ionic strength of the release medium may cause dissociation of DOX $/ \gamma$-PGA complex.

Effect of Ionic Strength on DOX Release from DOX/ $\gamma-P G A$ Ionic Complexes. The in vitro DOX release profiles from ionic complex $(1: 1 \mathrm{w} / \mathrm{w})$ suspended in phosphate buffered saline ( $\mathrm{pH}$ 7.4) at different ionic strengths namely; $77 \mathrm{mM}$, $154 \mathrm{mM}$, and $308 \mathrm{mM}$ are presented in Figure 8. Increasing the ionic strength of the release medium did not have a significant effect on the DOX release rate. At higher ionic strength (308 mM PBS), a maximum of $14 \%$ of total DOX was dissociated from the complex over 10-hour period, then DOX concentration decreases, probably due to its degradation in presence of high salt concentration. In case of low ionic strength buffer, only $8 \%$ of total drug were dissociated; however, under physiological buffer conditions (154 mM PBS, pH 7.4), DOX release was linear, releasing upto $15 \%$ of total DOX over 30 -hour period. Since the initial binding of drug to the polymer is predominantly based on electrostatic complementarity, increased ionic strength, to some extent, helps in mitigating the electrostatic attraction, resulting in less effective binding and hence relatively faster dissociation of drug from drug/polymer complex.

3.4. $p H$-Triggered Release of DOX from DOX/ $\gamma$-PGA Ionic Complexes. The in vitro DOX release from DOX/ $\gamma$-PGA complex at varying $\mathrm{pH}$ is shown in Figure 9. At $\mathrm{pH}$ 2.2, burst release of DOX was observed, releasing $40 \%$ of drug in the first 8 hours, followed by slow release over a week. No such burst release was observed at pH higher than 2.2. There could be several reasons for such a burst release of DOX in low $\mathrm{pH}$ environment. One possibility is that at $\mathrm{pH}$ 2.2 , most of the carboxyl groups in $\gamma$-PGA $\left(\mathrm{pK}_{\mathrm{a}}=2.6\right)$ are protonated, and so is the amine group in DOX $\left(\mathrm{NH}_{3}^{+}\right)$. Thus, the interaction between $\gamma$-PGA and DOX is weakened

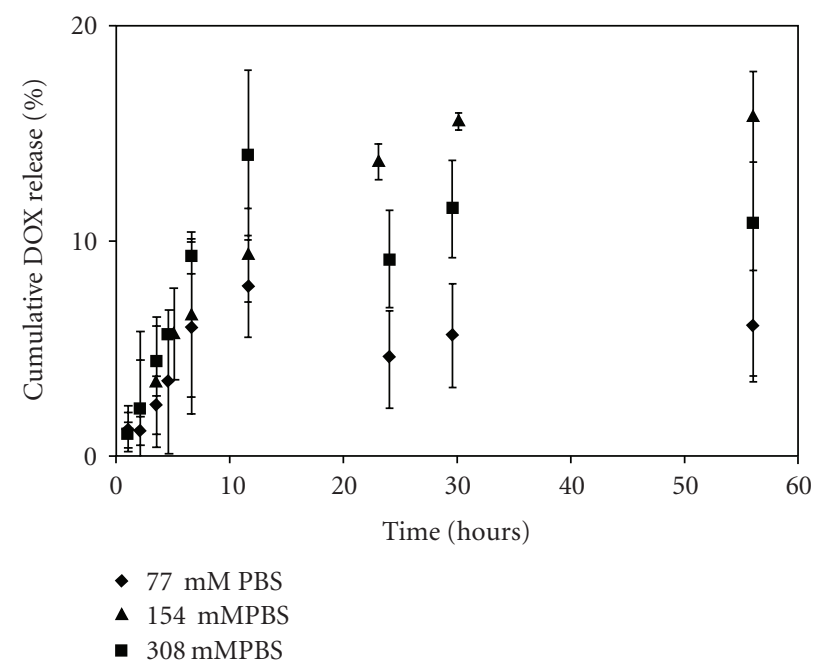

FIGURE 9: Effect of ionic strength on release of DOX from DOX/PGA ionic complex $(1: 1 \mathrm{w} / \mathrm{w})$ in phosphate buffered saline, $\mathrm{pH} 7.4$ at different ionic strengths.

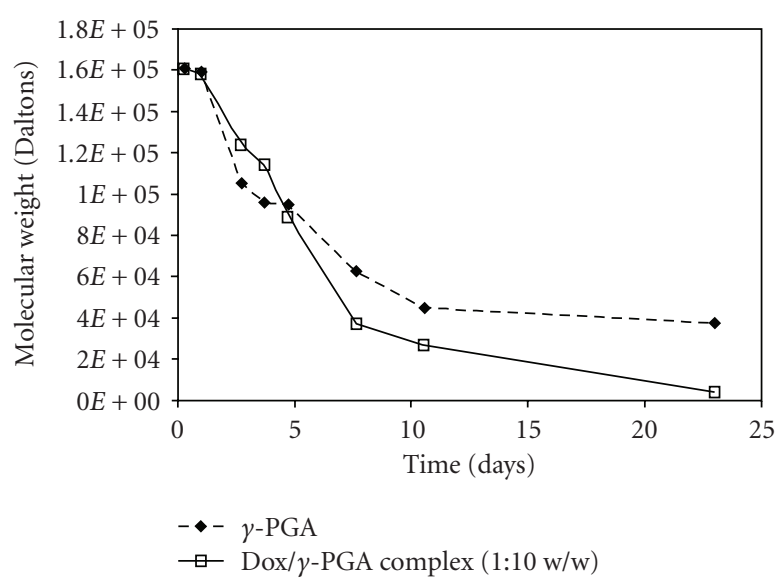

FIGURE 10: Degradation of $\gamma$-PGA and DOX $/ \gamma$-PGA ionic complex $(1: 10 \mathrm{w} / \mathrm{w})$ in $154 \mathrm{mM}$ phosphate buffered saline, $\mathrm{pH} 7.4$ at $37^{\circ} \mathrm{C}$.

because of electrostatic repulsion between both the positively charged drug and the polymer, thereby dissociating the drug.

Another possible reason could be the precipitation of $\gamma$ PGA under acidic conditions. At pH 2.2, a small fraction of $\gamma$-PGA precipitates out of the complex (visual observation), leaving behind the dissociated DOX in the dialysis bag, which then rapidly diffuses out into the release medium.

Based on these observations, one might assume a 100\% release of DOX at $\mathrm{pH}$ 2.2; however, DOX dissociation slows down after several hours. The possible explanation of such a phenomenon could be derived from thermal analysis of the complex. The DSC and TGA thermograms show that DOX association with $\gamma$-PGA is quite strong, which suggests the involvement of additional interactions between DOX and $\gamma$ PGA, apart from electrostatic forces. As a result, only a part of $\mathrm{DOX} / \gamma$-PGA ionic complex responded to $\mathrm{pH}$ change, leaving the rest of the complex undissociated, which dissociates at a much slower rate. 
On the other hand, at higher pHs (4 to 7.4), $\gamma$-PGA remains soluble, thus the only possible way that DOX can be released is by $\mathrm{pH}$-induced dissociation, thus no burst release effect is observed at any of the higher $\mathrm{pHs}$ studied. Nevertheless, in the $\mathrm{pH}$ range of 4-7.4, the carboxyl groups in $\gamma$-PGA are unprotonated; thus higher the $\mathrm{pH}$, more the number of un-protonated carboxyl groups, and stronger the polymer/drug interaction. Therefore, increasing the medium $\mathrm{pH}$ reduced the DOX dissociation, hence release rate. Up to $15 \%$ of total drug were released at the physiological $\mathrm{pH}$ as opposed to $50 \%$ and $30 \%$ at $\mathrm{pH} 4$ and 6 , respectively. From clinical perspective, it is desirable to achieve slow or minimal drug release from targeted drug delivery system at physiological $\mathrm{pH}$ in blood circulation thus reducing the drug loss until the intended target is reached. In all cases, the drug release from the complex was linear as observed over a week. It is thus concluded that $\mathrm{pH}$-triggered DOX $/ \gamma$-PGA complexes facilitate sustained release of DOX over a long period of time.

3.5. Degradation of DOX/ $\gamma$-PGA Ionic Complex. The biodegradability of $\gamma$-PGA and DOX $/ \gamma$-PGA complex was estimated following hydrolysis under physiological condition. As illustrated in Figure 10, the molecular weight of both $\gamma$-PGA and DOX $/ \gamma$-PGA ionic complex decreased exponentially from $170 \mathrm{kDa}$ to 40 and $\sim 10 \mathrm{kDa}$, respectively, over a period of 3 weeks. Since the molecular weight cut off of kidney is $\sim 70 \mathrm{kDa}$, thus both, the DOX-free $\gamma$-PGA and the undissociated DOX $/ \gamma$-PGA complex, can be excreted out by kidney within 2-3 weeks. However, detailed in vivo studies need to be performed to confirm the polymer degradation and renal clearance.

\section{Conclusions}

In this paper, we describe the feasibility of using $\gamma$-PGA as a carrier for the delivery of small, cationic anthracycline drug, DOX. We have demonstrated that DOX can interact with $\gamma$-PGA to form stable complexes with almost $100 \%$ complexation of DOX with $\gamma$-PGA. The polymer/drug complexation approach binds the drug tightly to the polymer; as a result, the formed complexes demonstrated $\mathrm{pH}$-dependent slow release of Doxorubicin, in vitro. More studies need to be done to prove the exact mechanism of interaction between DOX and $\gamma$-PGA. A detailed in vitro cytotoxicity studies are ongoing to determine the bioactivity of release of DOX and the effect of plasma proteins on DOX release from the ionic complex.

\section{Acknowledgments}

The authors sincerely thank their colleague and department's lab supervisor Mr. Brian Dennis for his technical assistance and discussion. This research was supported by the Natural Sciences and Engineering Research Council of Canada through Discovery Grant no. 4388 awarded to Dr. A. Margaritis.

\section{References}

[1] S. Feng and S. Chien, "Chemotherapeutic engineering: application and further development of chemical engineering principles for chemotherapy of cancer and other diseases," Chemical Engineering Science, vol. 58, pp. 4087-4114, 2003.

[2] S. M. Moghimi, A. C. Hunter, and J. C. Murray, "Longcirculating and target-specific nanoparticles: theory to practice," Pharmacological Reviews, vol. 53, pp. 283-318, 2001.

[3] B. Manocha and A. Margaritis, "Production and characterization of $\gamma$-polyglutamic acid nanoparticles for controlled anticancer drug release," Critical Reviews in Biotechnology, vol. 28, pp. 83-99, 2008.

[4] D. D. Von Hoff, M. Rozencweig, and M. Piccart, "The cardiotoxicity of anticancer agents," Seminars in Oncology, vol. 9, pp. 23-33, 1982.

[5] P. Singal, N. Iliskovic, T. Li, and D. Kumar, "Adriamycin cardiomyopathy: pathophysiology and prevention," FASEB Journal, vol. 11, pp. 931-936, 1997.

[6] L. J. Goldstein, H. Galski, A. Fojo, et al., "Expression of a multidrug resistance gene in human cancers," Journal of the National Cancer Institute, vol. 81, pp. 116-124, 1989.

[7] K. A. Janes, M. P. Fresneau, A. Marazuela, A. Fabra, and M. J. Alonso, "Chitosan nanoparticles as delivery systems for doxorubicin," Journal of Controlled Release, vol. 73, pp. 255267, 2001.

[8] D. Kalaria, G. Sharma, V. Beniwal, and M. Ravi Kumar, "Design of biodegradable nanoparticles for oral delivery of doxorubicin: in vivo pharmacokinetics and toxicity studies in rats," Pharmaceutical Research, vol. 26, pp. 492-501, 2009.

[9] A. Papagiannaros, S. Hatziantoniou, K. Dimas, G. T. Papaioannou, and C. Demetzos, "A liposomal formulation of Doxorubicin, composed of hexadecylphosphocholine (HePC): physicochemical characterization and cytotoxic activity against human cancer cell lines," Biomedicine \& Pharmacotherapy, vol. 60, pp. 36-42, 2006.

[10] S. Dreis, F. Rothweiler, M. Michaelis, J. Cinatl Jr., J. Kreuter, and K. Langer, "Preparation, characterisation and maintenance of drug efficacy of doxorubicin-loaded human serum albumin (HSA) nanoparticles," International Journal of Pharmaceutics, vol. 341, pp. 207-214, 2007.

[11] A. Gabizon, R. Shiota, and D. Papahadjopoulos, "Pharmacokinetics and tissue distribution of doxorubicin encapsulated in stable liposomes with long circulation times," Journal of the National Cancer Institute, vol. 81, pp. 1484-1488, 1989.

[12] D. Missirlis, R. Kawamura, N. Tirelli, and J. A. Hubbell, "Doxorubicin encapsulation and diffusional release from stable, polymeric, hydrogel nanoparticles," European Journal of Pharmaceutical Sciences, vol. 29, pp. 120-129, 2006.

[13] G. S. Kwon, M. Naito, M. Yokoyama, T. Okano, Y. Sakurai, and K. Kataoka, "Physical entrapment of adriamycin in AB block copolymer micelles," Pharmaceutical Research, vol. 12, pp. 192-195, 1995.

[14] P. Couvreur, B. Kante, L. Grislain, M. Roland, and P. Speiser, "Toxicity of polyalkylcyanoacrylate nanoparticles. II: doxorubicin-loaded nanoparticles," Journal of Pharmaceutical Sciences, vol. 71, pp. 790-792, 1982.

[15] K. Akiyoshi, I. Taniguchi, H. Fukui, and J. Sunamoto, "Hydrogel nanoparticle formed by self-assembly of hydrophobized polysaccharide. Stabilization of adriamycin by complexation," European Journal of Pharmaceutics and Biopharmaceutics, vol. 42, pp. 286-290, 1996. 
[16] X. R. Teng, D. G. Shchukin, and H. Möhwald, "A novel drug carrier: lipophilic drug-loaded polyglutamate/ polyelectrolyte nanocontainers," Langmuir, vol. 24, pp. 383-389, 2008.

[17] P. Vishweshwar, J. A. McMahon, J. A. Bis, and M. J. Zaworotko, "Pharmaceutical co-crystals," Journal of Pharmaceutical Sciences, vol. 95, pp. 499-516, 2006.

[18] A. Jimenez-Kairuz, D. A. Allemandi, and R. H. Manzo, "The improvement of aqueous chemical stability of a model basic drug by ion pairing with acid groups of polyelectrolytes," International Journal of Pharmaceutics, vol. 269, pp. 149-156, 2004.

[19] B. Guo, J. Yuan, and Q. Gao, "pH and ionic sensitive chitosan/carboxymethyl chitosan IPN complex films for the controlled release of coenzyme A," Colloid and Polymer Science, vol. 286, pp. 175-181, 2008.

[20] C. Agresti, Z. Tu, C. Ng, Y. Yang, and J. F. Liang, "Specific interactions between diphenhydramine and $\alpha$-helical poly(glutamic acid) - a new ion-pairing complex for taste masking and $\mathrm{pH}$-controlled diphenhydramine release," European Journal of Pharmaceutics and Biopharmaceutics, vol. 70, pp. 226-233, 2008.

[21] C. Li, "Poly(L-glutamic acid)—anticancer drug conjugates," Advanced Drug Delivery Reviews, vol. 54, pp. 695-713, 2002.

[22] J. M. Buescher and A. Margaritis, "Microbial biosynthesis of polyglutamic acid biopolymer and applications in the biopharmaceutical, biomedical and food industries," Critical Reviews in Biotechnology, vol. 27, pp. 1-19, 2007.

[23] B. Manocha and A. Margaritis, "A novel method for the selective recovery and purification of $\gamma$-polyglutamic acid from Bacillus licheniformis fermentation broth," Biotechnology Progress, vol. 26, no. 3, 2010.

[24] S. R. Urva, B. S. Shin, V. C. Yang, and J. P. Balthasar, "Sensitive high performance liquid chromatographic assay for assessment of doxorubicin pharmacokinetics in mouse plasma and tissues," Journal of Chromatography B, vol. 877, pp. 837$841,2009$.

[25] R. Khachatoorian, I. G. Petrisor, and T. F. Yen, "Prediction of plugging effect of biopolymers using their glass transition temperatures," Journal of Petroleum Science and Engineering, vol. 41, pp. 243-251, 2004.

[26] G. Ho, T. Ho, K. Hsieh, et al., " $\gamma$-Polyglutamic acid produced by Bacillus subtilis (natto): structural characteristics, chemical properties and biological functionalities," Journal of the Chinese Chemical Society, vol. 53, pp. 1363-1384, 2006. 

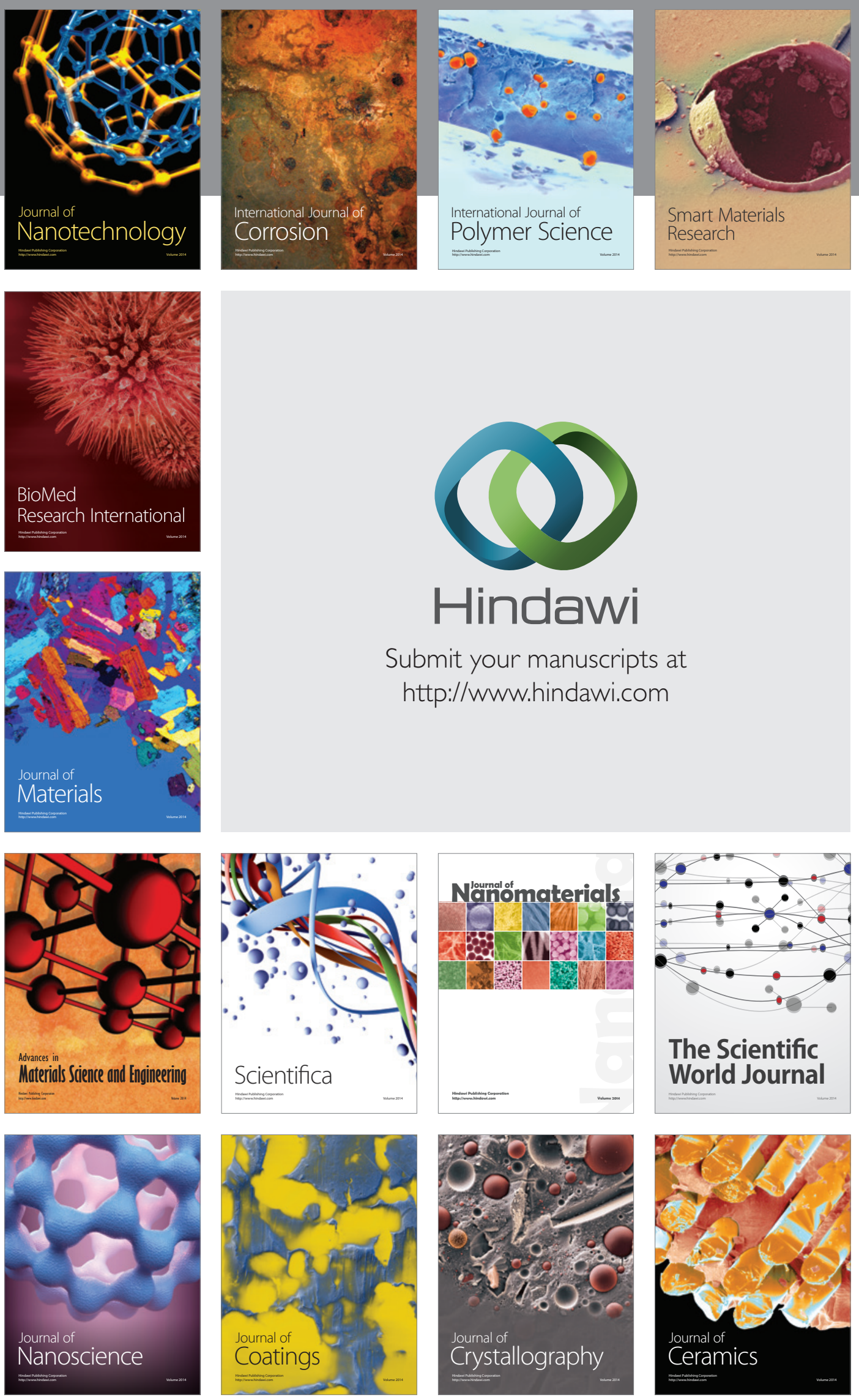

The Scientific World Journal

Submit your manuscripts at

http://www.hindawi.com

\section{World Journal}

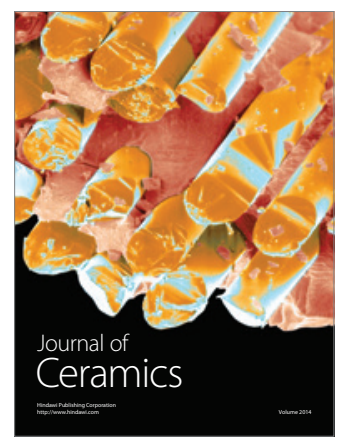

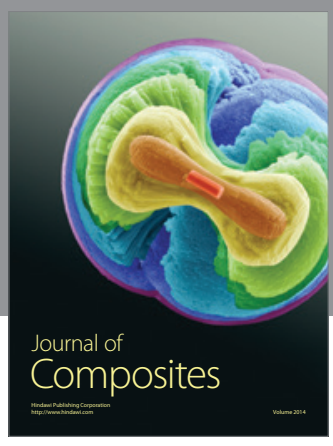
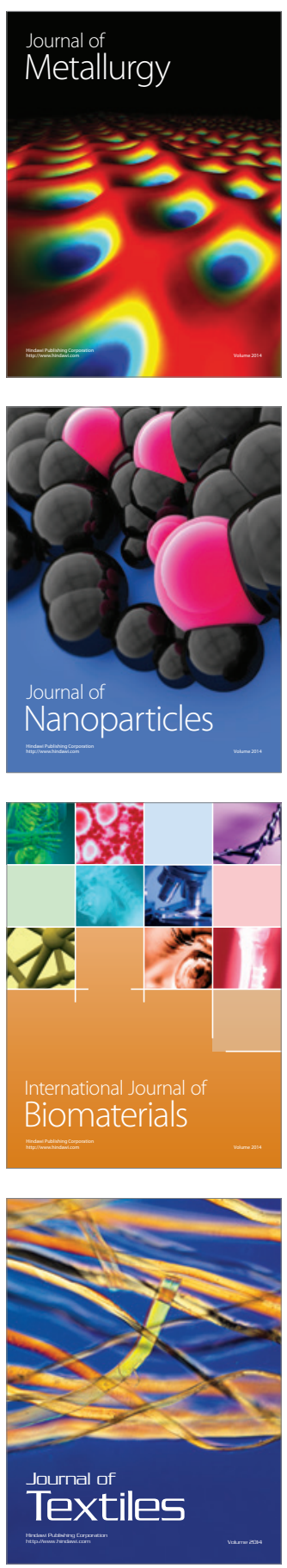